\title{
Cooperative Compressed Sensing schemes for Telemonitoring of Vital Signals in WBANs
}

\author{
Aris S. Lalos*, Elli Kartsakli*, Angelos Antonopoulos ${ }^{\dagger}$, Stefano Tennina ${ }^{\ddagger}$, \\ Marco Di Renzo ${ }^{\S}$, Luis Alonso* and Christos Verikoukis ${ }^{\dagger}$ \\ * Department of Signal Theory and Communications (TSC), Technical University of Catalonia, Barcelona Spain \\ $\dagger$ Telecommunications Technological Center of Catalonia (CTTC), Castelldefels, Spain \\ $\ddagger$ WEST Aquila srl, L’Aquila, Italy \\ $\S$ Supelec CNRS, Paris, France \\ Email: \{aristeidis.lalos, ellik, luisg\}@tsc.upc.edu, \{aantonopoulos, cveri\}@cttc.es, \\ tennina@westaquila.it,marco.direnzo@lss.supelec.fr
}

\begin{abstract}
Wireless Body Area Networks (WBANs) are composed of various sensors that either monitor and transmit real time vital signals or act as relays that forward the received data packets to a nearby Body Node Coordinator (BNC). The design of an accurate and energy efficient wireless telemonitoring system can be achieved by: i) minimizing the amount of data that should be transmitted for an accurate reconstruction at the $\mathrm{BNC}$, and ii) increasing the robustness of the telemonitoring system to link failures due to the nature of wireless medium. To this end, we present a novel Compressed Sensing (CS) based telemonitoring scheme, called Cooperative Compressed Sensing (CCS), that exploits the benefits of Random Linear Network Coding (RLNC) along with key characteristics of the transmitted biosignals in order to achieve an energy efficient signal reconstruction at the BNC. Simulation studies, carried out with real electrocardiographic (ECG) data, show the benefits of: i) employing RLNC, compared to the case where relays simply store and forward the original data packets, and ii) applying the proposed CCS scheme, compared to traditional CS recovery approaches.
\end{abstract}

\section{INTRODUCTION}

Wireless Body Area Networks (WBANs) are consisting of a collection of low-power, lightweight wireless sensor nodes, either wearable or implantable, highly specialized to perform a specific task. More precisely, these sensors are either employed: i) for continuously monitoring human's physiological activities and actions [1] or ii) as relays that help other body sensors to transmit their information [2], to a nearby Body Node Coordinator (BNC) (e.g., smartphone) via ultra-low-power short range radios (e.g., ZigBee, low energy (LE) Bluetooth).

Apart from the obvious advantages, wireless telemonitoring of vital signals (e.g., electromyography (EMG), electroencephalography (EEG), electrocardiography (ECG), etc.) requires new schemes and algorithms to be implemented in order to optimize important parameters, such as: i) the energy consumption at the body node (sensor/relay) side and ii) the robustness of the telemonitoring systems in link failures that may occur due to significant path loss [2], effects of postural body movements relative to sitting, walking, running [3] or interference between multiple closely located WBANs [4]. According to [5], the $\mathrm{RF}$ power amplifier of a WBAN transmitter consumes $73 \%$ of the total power, while the pervasive use of WBAN increases the need for coexistence of multiple WBANs (e.g., patients with attached body nodes in a medical center). Consequently, the efficient compression, transmission and reconstruction of the information constitutes a major challenge in WBANs.

The compression/reconstruction efficiency of the algorithms running on nodes in the network can be optimized by proposing encoding/decoding schemes, with high Compression Ratio (CR) capabilities and reduced computational requirements. To this end, Compressed Sensing (CS) approaches for signal compression/reconstruction offer an affordable solution. CS provides a way of reconstructing a sparse signal from a small number of random linear combinations. Several works [5], [6] assume that the compression of the ECG or EMG signals can be efficiently implemented at the biosensor (source) by simply performing Random Linear Coding (RLC) of the recorded measurements, while efficient reconstruction is achieved by applying default CS algorithms (e.g., LASSO) that exploit sample sparsity at the receiver. Zhang et al. [7], first proposed a scheme that improves the CR capabilities of the default CS algorithms by exploiting key characteristics (e.g., block sparse structure) of the transmitted signals. However the main drawback of the proposed scheme was the increased computational recovery requirements.

All the aforementioned works assume direct communication scenarios, completely ignoring the fact that body nodes are often able to communicate with each other. Therefore, the communication efficiency of the aforementioned telemonitoring schemes would greatly benefit from the application of Random Linear Network Coded (RLNC) cooperation. The main idea behind RLNC cooperation is that intermediate relays can encode input data packets using simple algebraic operations, before forwarding them to the neighboring nodes. Hence, coupling RLNC and CS can lead to significant efficiency gains in WBANs-based 
telemonitoring applications. To the best of our knowledge, this is the first work that demonstrate the benefits of coupling source (RLC) and network (RLNC) coding schemes for the efficient telemonitoring of vital signals in WBANs. Moreover, the benefits of the proposed framework, called Cooperative Compressed Sensing (CCS), are further enhanced by taking into account specific key characteristics (e.g., high temporal correlation, block sparsity) of the recorded measurements (e.g., ECG, EEG, EMG) during the signal reconstruction. To that end, we also propose a novel low complexity recovery algorithm, named Decorrelated Iterative reweighed Group LASSO (DIG LASSO), that exploits the block sparsity of the decorrelated signal to provide the network with robustness to link failures and higher energy efficiency, compared to traditional CS recovery schemes.

The paper is outlined as follows: Section II, introduces the concepts and terminology related with the CS theory. Section III presents the system model and Section IV describes the operations that can be carried out at the relay node. In Section V, we introduce the proposed recovery algorithm (DIG LASSO). In Section VI, the proposed scheme is evaluated and compared against the state-ofthe-art approaches.

\section{Preliminaries on Compressed Sensing}

CS provides a way of reconstructing a sparse signal $\mathbf{x} \in$ $\mathbb{R}^{N}$ by using only a small number of linearly combined measurements [8], [9]. The RLC measurements $\mathbf{y} \in \mathbb{R}^{M}$, $M<N$, are generated using a random matrix $\mathbf{A} \in \mathbb{R}^{M \times N}$ with i.i.d. elements as: $\mathbf{y}=\mathbf{A} \mathbf{x}+\mathbf{w}$, where $\mathbf{w}$ is a vector with noise samples.

\section{A. Reconstruction by Exploiting Sample Sparsity}

In the noise free case (i.e., $\mathbf{w}=\mathbf{0}_{N}$ ), vector $\mathbf{x}$ may be ideally recovered from $\mathbf{y}$ by solving the problem: $\min _{x}\left\{\|\mathbf{x}\|_{0}: \mathbf{y}=\mathbf{A x}\right\}$, where $\|\cdot\|_{0}$ denotes the $\ell_{0}$ - norm that equals to the number of nonzero entries of $\mathbf{x}$.

In order for the signal reconstruction to be robust in the presence of noise, the constraint of the problem is relaxed: $\min _{x}\left\{\|\mathbf{x}\|_{0}:\|\mathbf{y}-\mathbf{A x}\|_{2}^{2} \leq \epsilon\right\}$, where $\epsilon$ is a predefined error tolerance and $\|\cdot\|_{2}$ is the $\ell_{2}-$ norm of the input vector, respectively. The above optimization problem cannot be used for practical applications, since it is computationally intractable. CS suggests replacing the $\ell_{0}$ quasi-norm by the convex $\ell_{1}$-norm and solving the following problem: $\min _{x}\left\{\|\mathbf{x}\|_{1}:\|\mathbf{y}-\mathbf{A x}\|_{2}^{2} \leq \epsilon\right\}$, where the $\ell_{1}$-norm, is defined as $\|\mathbf{x}\|_{1}=\sum_{i=1}^{N}\left|x_{i}\right|$. The $\ell_{1}$ minimization approach is a convex optimization problem that can be solved efficiently by linear programming techniques. By employing Langrange relaxation, we are able to efficiently approximate the solution of the aforementioned problem by solving the $\ell_{1}$ regularized least square problem:

$$
\hat{\mathbf{x}}:=\underset{\mathbf{x}}{\arg \min }\|\mathbf{y}-\mathbf{A x}\|_{2}^{2}+\lambda\|\mathbf{x}\|_{1}
$$

where the parameter $\lambda$ controls the balance between the two optimization objectives: i) the noise level $\|\mathbf{y}-\mathbf{A x}\|_{2}^{2}$ and ii) the sparsity of vector $\mathbf{x}$. Algorithmically, the convex optimization problem in eq. (1), known as LASSO problem, can be tackled by any generic second-order cone program (SOCP) solver.

\section{B. Reconstruction by Exploiting Block Sparsity}

A block sparse signal consists of clusters of zero and non - zero coefficients. To be more specific, vector $\mathbf{x}$ can be viewed as a concatenation of $R$ blocks of length $d$ :

$$
\mathbf{x}=[\underbrace{x_{1}, \ldots, x_{d}}_{\mathbf{x}^{T}[1]}, \underbrace{x_{d+1}, \ldots, x_{2 d}}_{\mathbf{x}^{T}[2]}, \ldots, \underbrace{x_{N-d+1}, \ldots, x_{N}}_{\mathbf{x}^{T}[R]}]^{T},
$$

where $\mathbf{x}[i]$ denotes the $i^{\text {th }}$ block and $N=R d$.

Similarly to eq. (2), we can represent matrix $\mathbf{A}$ as a concatenation of sub-matrices $\mathbf{A}[i]$ of size $M \times d$ : $\mathbf{A}=$ $\left[\begin{array}{llll}\mathbf{A}[1] & \mathbf{A}[2] & \ldots & \mathbf{A}[R]\end{array}\right]$. It has been shown that the block sparse structure enables the signal recovery from a reduced number of samples, compared to sparse structures. By simply using the $\ell_{1}$ relaxation for reconstructing $\mathbf{x}$, we ignore the fact that the signal is block-sparse, i.e., the non-zero entries occur in consecutive positions. To exploit block sparsity, we have to reconstruct vector $\mathbf{x}$ by solving:

$$
\hat{\mathbf{x}}:=\underset{\mathbf{x}}{\arg \min }\left\|\mathbf{y}-\sum_{i=1}^{R} \mathbf{A}[i] \mathbf{x}[i]\right\|_{2}^{2}+\sum_{i=1}^{R} \lambda_{i}\|\mathbf{x}[i]\|_{2},
$$

which is also known as group LASSO problem [10].

\section{Reconstruction by Exploiting Sparsity in a Transform} Domain

In many applications, although the signal $\mathbf{x}$ is not sparse at the time domain, it can be sparse in some transformed domains, such as the wavelet domain. Therefore, $\mathbf{x}$ can be expressed as $\mathbf{x}=\mathbf{W s}$, where $\mathbf{W} \in \mathbb{R}^{N \times N}$ is an orthonormal basis matrix of a transformed domain and $\mathbf{s}$ is the representation coefficient vector, which is sparse. In such cases, in order to exploit either the sample or the block sparsity of $\mathbf{s}$ in the transformed domain, instead of solving problems (1) or (3), we can solve:

$$
\begin{aligned}
& \hat{\mathbf{s}}:=\underset{\mathbf{s}}{\arg \min }\|\mathbf{y}-\mathbf{A W s}\|_{2}^{2}+\lambda\|\mathbf{s}\|_{1} \\
& \hat{\mathbf{s}}:=\underset{\mathbf{s}}{\arg \min }\|\mathbf{y}-\mathbf{A W s}\|_{2}^{2}+\sum_{i=1}^{R} \lambda_{i}\|\mathbf{s}[i]\|_{2}
\end{aligned}
$$

and then reconstruct $\hat{\mathbf{x}}=\mathbf{W} \hat{\mathbf{s}}$.

\section{Wireless Telemonitoring Model}

Figure 1 presents our system model, where we consider a WBAN, with a biosensor (source node) that records a real time vital signal (e.g., ECG or EEG), and transmits it to the BNC (i.e. smartphone) through an intermediate relay body node. We assume that all the sensors are attached on the same body and the packet error probability in each hop is denoted by $p_{e}$. 


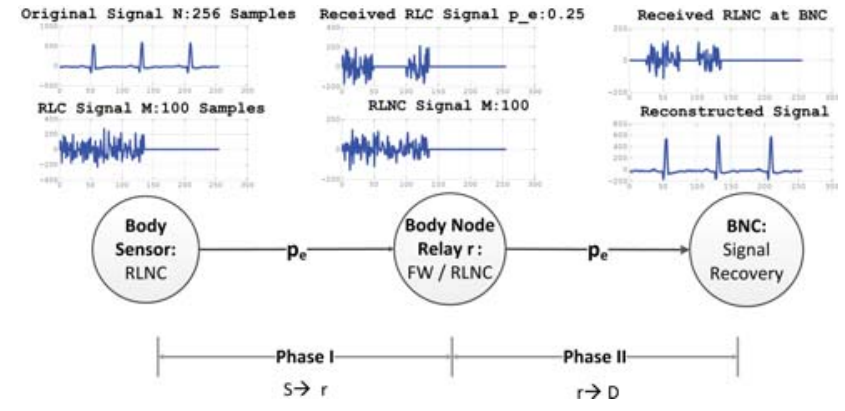

Fig. 1. Wireless Telemonitoring Model of a WBAN.

\section{A. Operations Executed at the Source}

The vital signal is divided into segments of $\mathrm{N}$ samples and each segment is represented as a vector $\mathbf{x}=$ $\left[x_{1}, \ldots, x_{N}\right]^{T}$, where $x_{i} \in \mathbb{R}$. We assume that the recorded signal to be transmitted contains noise itself and, as a result, it may be written as $\mathbf{u}=\mathbf{x}+\mathbf{w}_{s}$, where $\mathbf{u}=\left[u_{1}, \ldots, u_{N}\right]^{T}$ are samples of the noisy signal and $\mathbf{w}_{s}=\left[w_{1}, \ldots, w_{N}\right]^{T}$ is the random noise. For each segment, the source generates $M$ random linear combinations (performing RLC) by using a random matrix $\mathbf{A}$ of dimension $M \times N$, as follows: $\mathbf{y}=\mathbf{A} \mathbf{u}=\mathbf{A x}+\mathbf{w}$, where $\mathbf{y}=\left[y_{1}, \ldots, y_{M}\right]^{T}$ and $\mathbf{w}=\mathbf{A w}_{s}$. The coefficients $\mathbf{A}_{i, j}$ are selected from the Bernoulli distribution, i.e. $\mathbf{A}_{i, j}=$ $\pm 1 / \sqrt{N}$ with probability 0.5 .

\section{B. Communication Protocol $\&$ Network Energy Efficiency}

Each RLC measurement $y_{i}, i=1, \ldots, M$ is included in a packet and its transmission to the final sink node is performed in two phases. In Phase I $\left(P_{I}\right)$ the communication protocol is based on a Time Division mechanism where $\mathrm{M}$ time slots are assigned to the source node in order to transmit the RLC measurements. Given that the packet error probability is $p_{e}$, the relay receives on average $N_{r}=\left(1-p_{e}\right) M$ correct packets, assuming that the wrong packets are simply discarded. As a result, in Phase II $\left(P_{I I}\right)$ the relay node is capable either to forward the received $N_{r}$ measurements (FW policy) or to perform RLNC and transmit $M$ random linear combinations of the received measurements (RLNC policy). In the next section, we provide end-to-end expressions for both policies, with regard to the received measurements at the $\mathrm{BNC}$, given the original uncoded signal segment $\mathbf{x}$. For the evaluation of the two policies, we adopt the energy efficiency $(E E)$ metric, defined as:

$$
E E=\frac{\text { Reconstructed Data }}{\text { Total Energy }}=\frac{N l_{m}}{E_{P_{I}}+E_{P_{I I}}} \text { bits/Joule, }
$$

where $E_{P_{I}}, E_{P_{I I}}$ correspond to the average energy consumed during the first and second phase respectively, and $l_{m}$ denotes the length of each measurement in bits. For both policies, the energy $E_{P_{I}}$ is defined as:

$$
E_{P_{I}}=M^{*} E_{T}+M^{*} E_{R},
$$

where $E_{T}=P_{T} T_{s}, E_{R}=P_{R} T_{s}$ are the transmit and receive energy consumed by the network nodes in one slot of duration $T_{s}$. With $P_{T}$ and $P_{R}$ we denote the transmit and receive power. The number of transmitted data $M^{*}$ is the measurements required for ensuring an accurate ECG reconstruction at the $\mathrm{BNC}$ and depends on both the adopted relay policy and the reconstruction method used at the BNC. Regarding the FW policy, the energy consumed at Phase II is given by:

$$
E_{P_{I I}}^{F W}=N_{r}^{*} E_{T}+N_{r}^{*} E_{R}
$$

where $N_{r}^{*}=\left(1-p_{e}\right) M^{*}$. The respective energy consumption for the RLNC policy is given by:

$$
E_{P_{I I}}^{R L N C}=M^{*} E_{T}+M^{*} E_{R}
$$

\section{Relay Policies}

According to the communication protocol described above, the received measurements at the relay after $M$ transmissions may be written as:

$$
\mathbf{y}_{r}=\mathbf{I}_{e_{1}} \mathbf{y}=\mathbf{I}_{e_{1}} \mathbf{A x}+\mathbf{I}_{e_{1}} \mathbf{w}
$$

where $\mathbf{I}_{e_{1}}$ is an $N_{r} \times M$ matrix that directly occurs by selecting randomly $N_{r}=\left(1-p_{e}\right) M$ rows from the identity $\operatorname{matrix} \mathbf{I}_{M}$.

\section{A. Forwarding (FW) Policy}

In this case, the relay forwards $N_{r}$ received packets :

$$
\mathbf{x}_{\mathbf{F}} \mathbf{W}_{\mathbf{r}}=\mathbf{y}_{r}=\mathbf{I}_{e_{1}} \mathbf{A x}+\mathbf{w}_{r},
$$

where $\mathbf{w}_{r}=\mathbf{I}_{e_{1}} \mathbf{w}$. The received measurements at th destination may be written as in eq. (10):

$$
\begin{aligned}
\mathbf{y}_{F W_{d}} & =\mathbf{I}_{e_{2}} \mathbf{x}_{\mathbf{F} \mathbf{W}_{\mathbf{r}}}=\mathbf{I}_{e_{2}} \mathbf{I}_{e_{1}} \mathbf{A} \mathbf{x}+\mathbf{w}_{F W_{d}} \\
& =\mathbf{A}_{F W} \mathbf{x}+\mathbf{w}_{F W_{d}},
\end{aligned}
$$

where $\mathbf{A}_{F W}=\mathbf{I}_{e_{2}} \mathbf{I}_{e_{1}} \mathbf{A}$ and $\mathbf{w}_{F W_{d}}=\mathbf{I}_{e_{2}} \mathbf{I}_{e_{1}} \mathbf{w}$. Similarly to $\mathbf{I}_{e_{1}}, \mathbf{I}_{e_{2}}$ is an $\left(1-p_{e}\right) N_{r} \times N_{r}$ matrix that directly occurs by selecting randomly $\left(1-p_{e}\right) N_{r}$ rows from the identity matrix $\mathbf{I}_{N_{r}}$

\section{B. Random Linear Network Coding (RLNC) Policy}

In this case, the relay performs RLNC to the received measurements and forwards the packets:

$$
\mathbf{x}_{\mathbf{R L N C}_{\mathbf{r}}}=\mathbf{A}_{r} \mathbf{y}_{r}=\mathbf{A}_{r} \mathbf{I}_{e_{1}} \mathbf{A} \mathbf{x}+\mathbf{A}_{r} \tilde{\mathbf{w}}
$$

where $\mathbf{A}_{r}$ is an $M \times N_{r}$ matrix with Bernouli i.i.d. corefficients $\mathbf{A}_{r_{i, j}} \pm 1 / \sqrt{N_{r}}$ with probability 0.5. Thus, the received packets at the destination are given by:

$$
\begin{aligned}
\mathbf{y}_{R L N C_{d}} & =\mathbf{I}_{e_{3}} \mathbf{x}_{\mathbf{R L N C}}=\mathbf{I}_{e_{3}} \mathbf{A}_{r} \mathbf{I}_{e_{1}} \mathbf{A} \mathbf{x}+\mathbf{w}_{R L N C_{d}} \\
& =\mathbf{A}_{R L N C} \mathbf{x}+\mathbf{w}_{R L N C_{d}},
\end{aligned}
$$


with $\mathbf{A}_{R L N C}=\mathbf{I}_{e_{3}} \mathbf{A}_{r} \mathbf{I}_{e_{1}} \mathbf{A}$ and $\mathbf{w}_{R L N C_{d}}=\mathbf{I}_{e_{3}} \mathbf{A}_{r} \mathbf{I}_{e_{1}} \mathbf{w}$ Note that $\mathbf{I}_{e_{3}}$ is constructed in a similar way as $I_{e_{1}}$, from the identity matrix $\mathbf{I}_{M}$ after removing $p_{e} M$ randomly selected rows.

\section{Efficient Recovery of the Original Signal}

In real time sensing applications, the sensor observations are temporally correlated to a certain degree. In the following section, we propose a novel recovery algorithm that efficiently exploits the temporal signal correlations, enabling the original signal reconstruction by less received measurements. The proposed recovery algorithm, named decorelated iterative reweighted group LASSO (DIG LASSO) algorithm, will be derived in the two following subsections.

\section{A. Temporal Correlation-Aware Block Sparse Recovery}

Initially, we describe a novel efficient way of exploiting any possible temporal correlations between the elements of a signal block $\mathbf{x}[i]$, during block sparse recovery of the original signal. Let us assume that: i) the matrix $\mathbf{R}_{i} \in$ $\mathbb{R}^{d \times d}$ captures the correlation structure of the $i$-th block of sensor measurements, and ii) the correlation between elements of different signal blocks is zero, i.e.,

$$
E\left[\mathbf{x}[i] \mathbf{x}^{T}[j]\right]= \begin{cases}\mathbf{R}_{i} & \text { if } i=j \\ 0 & \text { if } i \neq j\end{cases}
$$

Each block correlation matrix $\mathbf{R}_{i}$ can be approximated by a Toeplitz symmetric matrix, given by:

$$
\mathbf{R}_{i}=\left[\begin{array}{cccc}
r_{0} & r_{1} & \ldots & r_{d-1} \\
r_{1} & r_{0} & \ldots & r_{d-2} \\
\vdots & \ddots & \ddots & \vdots \\
r_{d-1} & \ldots & r_{1} & r_{0}
\end{array}\right]
$$

The values of $r_{k}, k=0, \ldots, d-1$, can be estimated at the source by using an exponentially decaying sample window:

$$
r_{k}=a r_{k}+(1-a) \frac{1}{R} \sum_{i=1}^{R} \sum_{j=1}^{d-k} \frac{\mathbf{x}[i](j) \mathbf{x}[i](j+k)}{|\mathbf{x}[i](j)|^{2}}
$$

where $a$ is the selected forgetting factor and $\mathbf{x}[i](j)=$ $x_{(i-1) d+j}, j=1, \ldots, d, i=1, \ldots, R$ denotes the $\mathrm{j}$-th element of the i-th block. Alternatively, we can assume that intra-block correlation follows an exponential correlation model by making the approximation $r_{k}=r^{k}, k=$ $0, \ldots, d-1$ and selecting specific values for $r$ that capture the degree of correlation between adjacent samples.

Based on the fact that the group LASSO schemes become more efficient when the difference between the norms of non-zero blocks is small, we propose a practical way to achieve this (especially in highly correlated cases) by simply performing block sparse reconstruction of the decorrelated sensor segment $\mathbf{x}$, that is written as:

$$
\begin{aligned}
\mathbf{s} & =\mathbf{R}^{-1 / 2} \mathbf{x} \\
\mathbf{R}^{-1 / 2} & =\left[\begin{array}{cccc}
\mathbf{R}_{1}^{-1 / 2} & \mathbf{0}_{d} & \ldots & \mathbf{0}_{d} \\
\ldots & \ddots & \ddots & \ldots \\
\mathbf{0}_{d} & \ldots & \ldots & \mathbf{R}_{R}^{-1 / 2}
\end{array}\right]
\end{aligned}
$$

Consequently, by solving the problem defined in eq. (5) after selecting $\mathbf{W}=\mathbf{R}^{1 / 2}$ :

$$
\hat{\mathbf{s}}:=\underset{\mathbf{s}}{\arg \min }\left\|\mathbf{y}-\mathbf{A} \mathbf{R}^{1 / 2} \mathbf{s}\right\|_{2}^{2}+\sum_{i=1}^{R} \lambda_{i}\|\mathbf{s}[i]\|_{2},
$$

allows us to recover the original vector at the receiver, by controling the block sparsity of the decorrelated segment $\mathbf{s}$ that occurs from the original vector $\mathbf{x}$, by eliminating any temporal correlations within signal blocks (intra-block correlation).

\section{B. Enhancing Recovery Efficiency by Iterative Reweighting}

In the previous subsection we described a transform domain group LASSO scheme that exploits temporal correlation of the recorded signal at the source to achieve more accurate reconstruction at the sink node. In this subsection, we will describe techniques that will allow to further enhance the recovery efficiency, leading to significant improvement in the network lifetime and higher robustness to wireless link failures.

To further promote block sparsity of $\mathbf{s}$, the $\|\mathbf{s}[i]\|_{2}$ terms in eq. (21) can be replaced by $\log \left(\|\mathbf{s}[i]\|_{2}+\epsilon\right)$ for a small positive $\epsilon$, ending up to the non convex problem:

$$
\hat{\mathbf{s}}:=\underset{\mathbf{s}}{\arg \min }\left\|\mathbf{y}-\mathbf{A} \mathbf{R}^{1 / 2} \mathbf{s}\right\|_{2}^{2}+\sum_{i=1}^{R} \lambda_{i} \log \left(\|\mathbf{s}[i]\|_{2}+\epsilon\right),
$$

where matrix $\mathbf{R}$ defined by eqs. (17), (20) captures the per block temporal correlations and $\epsilon$ prevents the cost from tending to $-\infty$. The function in eq. (22) is concave, but since it is smooth w.r.t. $\mathbf{s}$, iterative linearization may be utilized to obtain a local minimum [11]. Specifically, let $\mathbf{s}^{(l)}$ denote a tentative solution in the $l$-th iteration. The first order approximation of $\log \left(\|\mathbf{s}[i]\|_{2}+\epsilon\right)$ yields:

$$
\log \left(\|\mathbf{s}[i]\|_{2}+\epsilon\right) \leq \log \left(\left\|\mathbf{s}^{(0)}[i]\right\|_{2}+\epsilon\right)+\frac{\|\mathbf{s}[i]\|_{2}-\left\|\mathbf{s}^{(0)}[i]\right\|_{2}}{\left\|\mathbf{s}^{(0)}[i]\right\|_{2}+\epsilon} .
$$

Following the majorization-minimization approach presented in [11], instead of minimizing the function that depends on the left-hand side, we can minimize the majorizing function that depends on the right-hand side of (23) and iterate. Then, the problem in eq. (22) can be driven to a stationary point by executing the iterations:

$$
\begin{aligned}
& \mathbf{s}^{(l)}:=\underset{s}{\arg \min }\left\|\mathbf{y}-\mathbf{A R}^{1 / 2} \mathbf{s}\right\|_{2}^{2}+\sum_{i=1}^{R} \lambda_{i} w_{i}^{(l)}\|\mathbf{s}[i]\|_{2}(24) \\
& w_{i}^{(l)}:=\left(\left\|\mathbf{s}^{(l-1)}[i]\right\|_{2}+\epsilon\right)^{-1}, \quad i=1, \ldots, R .
\end{aligned}
$$




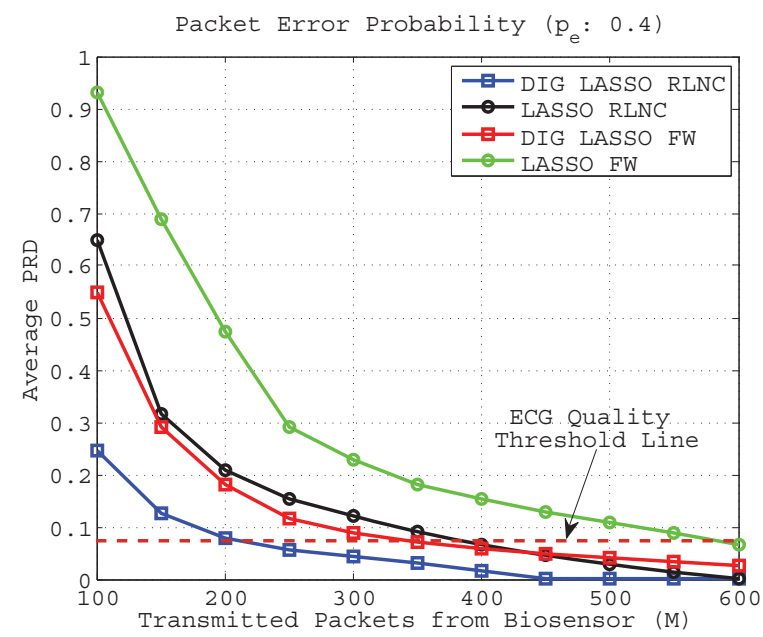

Fig. 2. Evaluation of different telemonitoring schemes in terms of the average PRD assuming a $p_{e}: 0.4$ error probability.

The optimization per iteration of eq. (24) is a weighted version of eq. (5), where $\mathbf{W}=\mathbf{R}^{-1 / 2}$ and, thus, can be efficiently solved by standard software packages. The iterations can be initialized with the solution of eq. (5), which corresponds to setting all weights to unity. The resulting iterative algorithm, so called as decorrelated iterative reweighted group LASSO (DIG LASSO), is terminated as soon as the relative error $\left\|\mathbf{s}^{(l)}-\mathbf{s}^{(l-1)}\right\|_{2} /\left\|\mathbf{s}^{(l)}\right\|_{2}$ becomes smaller than some predefined $\epsilon$ (e.g., $10^{-6}$ ). The original signal after $K$ required iterations is reconstructed as $\hat{\mathbf{x}}=\mathbf{R}^{1 / 2} \mathbf{S}^{(K)}$.

\section{Performance Evaluation}

The focus of this section is to identify the benefits of jointly applying the DIG LASSO and the two relay policies, in terms of both energy efficiency and robustness in link failures.

\section{A. ECG Dataset and Performance metrics}

For the evaluation of the proposed schemes we use the MIT-BIH Normal Sinus Rhythm Database [12]. We assume that the ECG signal is divided into segments of $N=256$ samples that correspond to a 2 sec ECG signal. Each segment is encoded at the biosensor side and the $M$ encoded RLC measurements are transmitted to a nearby relay body node. The relay is able either to simply forward (FW policy) or to perform RLNC and forward (RLNC policy) the re-encoded measurements to the destination. The BNC can reconstruct the original signal by performing either the default LASSO approach or our proposed algorithm (DIG LASSO). Therefore, we consider four different telemonitoring schemes denoted as: (a) DIG LASSO-RLNC, (b) LASSO-RLNC, (c) DIG LASSO-FW and (d) LASSO-FW. In each experiment, the aforementioned schemes are evaluated by using the same sensing matrices $\left(\mathbf{A}, \mathbf{A}_{r}\right)$ either to compress the

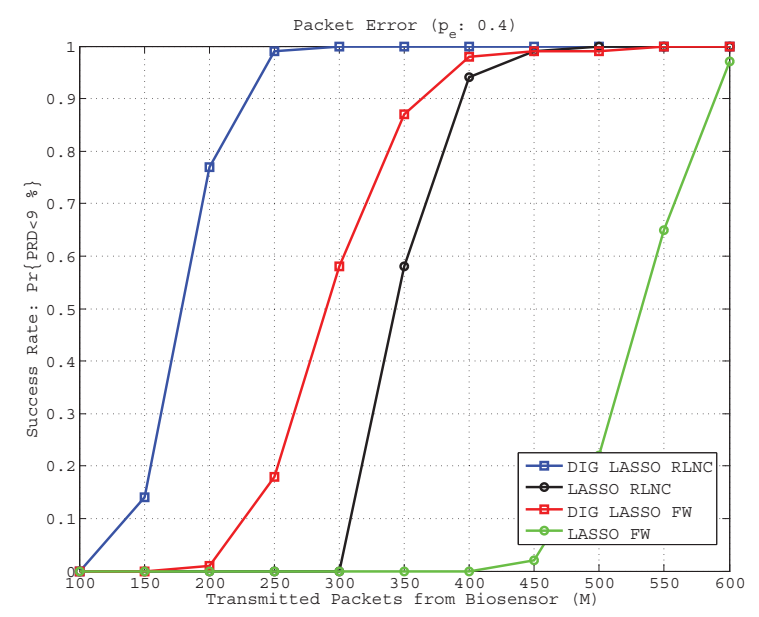

Fig. 3. Evaluation of different telemonitoring schemes in terms of the sucess rate assuming a $p_{e}: 0.4$ error probability.
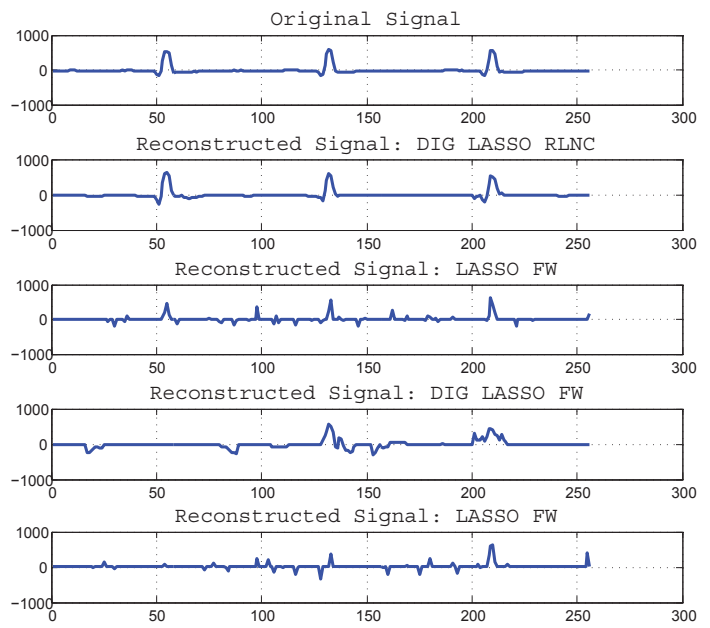

Fig. 4. Reconstruced Signals using the evaluated telemonitoring schemes for $M: 120$ and $p_{e}: 0.4$.

segments of the ECG recording at the biosensor (RLC) or to perform RLNC of the received measurement at the relay. In order to evaluate the diagnostic quality of the compressed ECG recordings, we employ the Percentage Root-mean-square Difference (PRD) [13] that is defined as: $P R D=\|\mathbf{x}-(\tilde{\mathbf{x}})\|_{2} /\|\mathbf{x}\|_{2} \times 100$, where $\mathbf{x}, \tilde{\mathbf{x}}$ denote the original and the reconstructed signal, respectively. In our scenarios, we assume that a reconstructed signal is acceptable at the BNC if and only if $P R D<9 \%$ [13]. Our goal is to evaluate the schemes (a) - (d) in terms of the network energy efficiency defined by eqs. (6) - (9).

\section{B. Performance Results}

In Fig.2, the obtained PRD, averaged over 1 h of 18 different ECG segments, is plotted against the $M$ transmitted RLC data by the biosensor. We assume a packet error rate equal to $p_{e}=0.4$. A block length $d=16$ was selected and the scaling rules for the parameter $\lambda$ in the problems 
TABLE I

ENERGY EFFICIENCY ( $10^{4}$ BITS/JOULE) OF WIRELESS TELEMONITORING SCHEMES, CASE STUDY: ECG

\begin{tabular}{|c||c|c|c|c|}
\hline & $\begin{array}{c}\text { DIG LASSO } \\
\text { RLNC }\end{array}$ & $\begin{array}{c}\text { LASSO } \\
\text { RLNC }\end{array}$ & $\begin{array}{c}\text { DIG LASSO } \\
\text { FW }\end{array}$ & $\begin{array}{c}\text { LASSO } \\
\text { FW }\end{array}$ \\
\hline \hline$p_{e}: 0.20$ & 2.84 & 1.65 & 2.18 & 1.58 \\
\hline$p_{e}: 0.40$ & 2.28 & 1.14 & 1.77 & 1.07 \\
\hline
\end{tabular}

(1) and (22) follow the results of [14]. By inspecting the figure, it is clear that the application of the DIG-LASSO algorithm reduces the number of the transmitted samples $M$ required for the efficient reconstruction of the signal $\mathbf{x}$, with respect to the reference schemes. Moreover, the application of RLNC at the intermediate node can further reduce the required number of transmissions.

Similar conclusions are drawn from fig. 3, where we plot the success rate as a function of the samples transmitted by the biosensor $M$. In particular, it is shown that the application of DIG LASSO-RLNC (case a) can lead to accurate reconstruction by transmitting only $M_{a}^{*}=225$ packets, whereas DIG LASSO-FW (case c) requires $M_{c}^{*}=$ 360 packets. The required number of packets for the reference schemes is significantly increased. In particular, LASSO-RLNC (case b) requires $M_{b}^{*}=450$, whereas at least $M_{d}^{*}=600$ packets are needed by LASSO-FW (case d). Thus, the application of RLNC at the relay results in a $22 \%$ reduction of the total packets required to be transmitted in the network ${ }^{1}$, while the application of the DIG-LASSO-RLNC, as compared to LASSO-RLNC, reduces the number of packet transmissions by $50 \%$. In Fig. 4, we plot the reconstructed ECG signal segments, that correspond to a 2 sec ECG recording. The number of samples transmitted by the biosensor was equal to $M=120$, and a packet error rate of $p_{e}=0.4$ was considered. Note that the DIG LASSO with RLNC at the relay provides the only accurate ECG signal reconstruction.

Finally, in Table I we provide the energy efficiency values according to eqs. (6) - (9) for different values for $p_{e}$. We have assumed that the transmit and receive power is equal to $3.8 \mathrm{~mW}, 4.6 \mathrm{~mW}$ respectively, while the duration of a

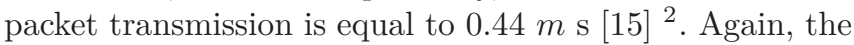
exploitation of temporal correlation during reconstruction and the application of RLNC at the relay body nodes result in significant energy efficiency gains, especially in cases with high link failures.

\section{ViI. Concluding Remarks}

Real time telemonitoring of vital signals in WBANs introduces several challenges, such as: i) minimizing the amount of data that should be transmitted in the WBAN

\footnotetext{
${ }^{1}$ In cases (a), (b) the total packet transmitted in the two phases $\left(P_{I}, P_{I I}\right)$ are $2 M_{a}^{*}$ and $2 M_{b}^{*}$ respectively. whereas in case $(\mathrm{c}),(\mathrm{d})$ the total pakcet are $\left(2-p_{e}\right) M_{c}^{*}$ and $\left(2-p_{e}\right) M_{d}^{*}$

${ }^{2}$ We have assumed packets of length 14 bytes, with 2 bytes payload and a data rate equal to $256 \mathrm{kbps}$ [3], while $l_{m}=16 \mathrm{bits} /$ measurement.
}

ii) increasing the robustness of the telemonitoring system to link failures. To this end, we proposed a novel Cooperative Compressed Sensing (CCS) framework that offers significant gains in terms of both energy efficiency of the nodes in the network and robustness to link failures, by exploiting the benefits of RLNC cooperation and the block sparse structure of the decorrelated biosignals during reconstruction. Although we focused on wireless ECG telemonitoring, the proposed schemes can be also applied to other telemedicine applications, such as telemonitoring of EEG [16] and EMG [5]. The presented schemes will be implemented in a real WSN testbed.

\section{ACKNOWLEDGMENTS}

This work has been partially funded by the Research Projects WSN4QoL (286047) and KINOPTIM (324491).

\section{REFERENCES}

[1] H. Cao, V. Leung, C. Chow, and H. Chan, "Enabling technologies for wireless body area networks: A survey and outlook," IEEE Commun. Mag., vol. 47, no. 12, pp. 84-93, 2009.

[2] Y. Chen et al. "Cooperative communications in ultra-wideband wireless body area networks: Channel modeling and system diversity analysis," IEEE J. Sel. Areas Commun, vol. 27, no. 1, pp. 5-16, January 2009

[3] S. Movassaghi et al., "Wireless body area networks: A survey," IEEE Commun. Surveys Tuts., vol. PP, no. 99, pp. 1-29, 2014

[4] J. Dong and D. Smith, "Opportunistic relaying in wireless body area networks: Coexistence performance," in IEEE Int. Conf. on Commun. (ICC), 2013, June 2013, pp. 5613-5618.

[5] A. Dixon et al., "Compressed sensing system considerations for ecg and emg wireless biosensors," IEEE Trans. Biomed. Circuits Syst., vol. 6, no. 2, pp. 156-166, 2012.

[6] H. Mamaghanian et al., "Compressed sensing for real-time energy-efficient ecg compression on wireless body sensor nodes," IEEE Trans. Biomed. Eng, vol. 58, no. 9, pp. 2456-2466, 2011.

[7] Z. Zhang et al., "Compressed sensing for energy-efficient wireless telemonitoring of noninvasive fetal ecg via block sparse bayesian learning," IEEE Trans. Biomed. Eng, vol. 60, no. 2, pp. 300-309, 2013.

[8] D. Donoho, "Compressed sensing," IEEE Trans. Inf. Theory, vol. 52, no. 4, pp. 1289-1306, 2006.

[9] E. Candes and T. Tao, "Near-optimal signal recovery from random projections: Universal encoding strategies?" IEEE Trans. Inf. Theory, vol. 52, no. 12, pp. 5406-5425, 2006.

[10] J. Friedman, T. Hastie, H. Hfling, and R. Tibshirani, "Pathwise coordinate optimization," Ann. Appl. Stat., Tech. Rep., 2007.

[11] D. Wipf and S. Nagarajan, "Iterative reweighted $\ell_{1}$ and $\ell_{2}$ methods for finding sparse solutions," IEEE Journal of Selected Topics in Signal Processing, vol. 4, no. 2, pp. 317-329, 2010.

[12] G. AL, A. LAN, G. L, H. JM, I. PCh, M. RG, M. JE, M. GB, P. C-K, and S. HE, "Physiobank, physiotoolkit, and physionet: Components of a new research resource for complex physiologic signals," Circulation, vol. 100, no. 23, pp. e215-e220, Jun 2013.

[13] Y. Zigel, A. Cohen, and A. Katz, "The weighted diagnostic distortion (wdd) measure for ecg signal compression," IEEE Trans. Biomed. Eng, vol. 47, no. 11, pp. 1422-1430, 2000.

[14] D. Angelosante, J. Bazerque, and G. Giannakis, "Online adaptive estimation of sparse signals: Where rls meets the $\ell_{1}$-norm." IEEE Trans. Signal Process, vol. 58, no. 7, pp. 3436-3447, 2010.

[15] LAN/MAN Standards Committee of the IEEE Computer Society, "IEEE Standard for Local and metropolitan area networks - Part 15.6: Wireless Body Area Networks," IEEE Std 802.15.62012, Feb. 2012.

[16] Z. Zhang, T.-P. Jung, S. Makeig, and B. Rao, "Compressed sensing of eeg for wireless telemonitoring with low energy consumption and inexpensive hardware," IEEE Trans. Biomed. Eng, vol. 60, no. 1, pp. 221-224, 2013. 